\title{
EFEITO ANTAGÔNICO DO FERRO E DO ZINCO EM UMA FORMULAÇÃO DE ALIMENTAÇÃO ENTERAL UTILIZANDO PLANEJAMENTO DE MISTURAS DA METODOLOGIA DE SUPERFÍCIE DE RESPOSTA
}

\author{
Luciana Bueno \\ Departamento de Alimentos e Nutrição Experimental, Divisão de Ciência dos Alimentos, Faculdade de Ciências Farmacêuticas, \\ Universidade de São Paulo, Av. Lineu Prestes, 580, 05508-900 São Paulo - SP, Brasil \\ Recebido em 15/5/07; aceito em 20/9/07; publicado na web em 26/2/08
}

\begin{abstract}
THE ANTAGONIC EFFECT OF IRON AND ZINC IN FORMULATIONS OF FEEDING DIETS STUDIED BY RESPONSE SURFACE METHODOLOGY FOR MIXTURES. Nutritional therapy with enteral diets became substantially specialized over the last years. This work's aim was to study the effect of the components of a formulation: fiber, calcium and medium-chain triglycerides for dialysability of minerals. Analysis of multiple variables was carried out using response surface methodology. The level curve showed an antagonic effect of interaction between iron and zinc. TCM was the variable responsible for characterizing competition between the two minerals in the formulation. More studies on foods are needed for knowing the effecs of minerals in formulations.
\end{abstract}

Keywords: iron; zinc; availability.

\section{INTRODUÇÂO}

A terapia nutricional enteral tem o objetivo de oferecer o aporte de nutrientes para indivíduos incapacitados ou impossibilitados de receber alimentação por via oral, visando manter ou recuperar as funções metabólicas. As determinações das possíveis interações entre nutrientes de formulações enterais possibilitam melhoria da qualidade e da eficiência da sua utilização na prática clínica. Desta forma, é possível potencializar o fornecimento de nutrientes direcionando o tratamento para necessidades específicas de pessoas doentes. ${ }^{1-6}$

Os minerais são nutrientes essenciais para a realização de mais de cem processos enzimáticos, além de exercerem funções fisiológicas no organismo humano. Entretanto, eles tendem a sofrer interações que determinam alterações na sua absorção, interferindo na qualidade nutricional das dietas enterais. A biodisponibilidade de minerais é geralmente definida pela medida da proporção do total do elemento contido no alimento, refeição ou dieta que é utilizado para a manutenção normal das funções do organismo. ${ }^{1-6}$

A estrutura química das fibras que contêm fitatos e oxalatos, por exemplo, interfere na disponibilidade do ferro e do zinco em dietas e alimentos, o que aumentaria a possibilidade de prejudicar o aproveitamento dos dois minerais. Altas quantidades de cálcio nos alimentos e dietas tendem a ser prejudiciais para a biodisponi-bilidade de ferro e zinco, devido às interações entre os minerais..$^{2-6}$ Os triacilglicerídeos de cadeia média (TCMs) não requerem a digestão de sais biliares e da lipase pancreática, sendo absorvidos diretamente no trato gastrintestinal. Tais produtos muitas vezes são adicionados às formulações enterais para aumentarem o aporte energético para indivíduos com distúrbio da absorção de lipídeos, devido à subnutrição grave. ${ }^{6-9}$

A biodisponibilidade de minerais presentes em alimentos e dietas tem sido determinada por métodos in vitro $e$ in vivo. Para o ferro e o zinco, existe correlação significativa entre os métodos, visto que os métodos "in vitro" reproduzem as condições do sistema digestório humano e são capazes de predizer os mecanismos de absorção de nutrientes. ${ }^{7-10}$

\footnotetext{
*e-mail: lubuenno@yahoo.com.br
}

Este trabalho teve como objetivo estudar as interações dos triglicerídeos de cadeia média (TCMs), da fibra e do cálcio na dialisabilidade do ferro e do zinco na maximização de uma formulação de alimentação enteral simultânea para os dois minerais pelo método in vitro e mostrar o antagonismo entre os dois minerais na formulação.

\section{PARTE EXPERIMENTAL}

\section{Material}

Foi selecionada a formulação de alimentação enteral prescrita para pacientes internados no Hospital Universitário da Universidade de São Paulo. A formulação foi reproduzida em laboratório com as respectivas matérias-primas que compunham a dieta selecionada, conforme mostrado na Tabela 1.

Os ingredientes para a formulação da dieta experimental foram obtidos de módulos comercializados. Utilizou-se isolado protéico de soja, maltodextrina, óleos de canola, de milho e TCM, sais minerais (Tabela 2) e mistura vitamínica.

As sete dietas preparadas, de acordo com o delineamento experimental utilizado para estudos centróide simplex de misturas de três componentes, possibilitando o estudo dos nutrientes escolhidos, foram manipuladas segundo consta na Tabela 3 .

O óleo de milho e a maltodextrina foram utilizados para complementar as dietas, mantendo-se o mesmo aporte calórico. Este procedimento permitiu que as sete dietas recomendadas no delineamento "centróide-simplex" mantivessem a mesma proporção de diluição final $(232,4 \mathrm{~g}$ de pó para 767,6 g de água) e aproximadamente o mesmo valor calórico (kcal=1.011,0/kg).

\section{Análises Físico-Químicas}

\section{Procedimentos analíticos}

Os procedimentos analíticos foram realizados segundo as normas propostas pela AOAC, ${ }^{11}$ com amostras em duplicatas ou triplicatas, utilizando-se a ração à base de caseína AIN - 93G, ${ }^{12}$ como padrão de referência secundário. 
Tabela 1. Dieta experimental

\begin{tabular}{|c|c|c|}
\hline Componentes & $\begin{array}{c}\text { Valores em } 100 \mathrm{~g} \\
\text { da mistura }\end{array}$ & $\begin{array}{l}\text { Valores em } 1 \mathrm{~L} \\
\text { de dieta diluída }\end{array}$ \\
\hline \multicolumn{3}{|l|}{ Proteína Total (g) } \\
\hline Isolado Protéico Soja ${ }^{1}$ & 13,34 & 31,00 \\
\hline \multicolumn{3}{|l|}{ Carboidrato Total (g) } \\
\hline Maltodextrina $^{2}$ & 59,12 & 137,40 \\
\hline \multicolumn{3}{|l|}{ Gordura $(\mathrm{g})$} \\
\hline Óleo de Canola & 7,74 & 18,00 \\
\hline Óleo de Milho & 5,38 & 12,50 \\
\hline $\mathrm{TCM}^{3}$ & 1,93 & 4,50 \\
\hline Lecitina de soja ${ }^{4}$ & 1,30 & 3,00 \\
\hline \multicolumn{3}{|l|}{ Minerais $(\mathrm{g})$} \\
\hline Mistura salina & 2,15 & 5,00 \\
\hline Carbonato de cálcio & 0,43 & 1,00 \\
\hline Vitaminas (g) & 4,30 & 10,00 \\
\hline \multicolumn{3}{|l|}{ Mistura vitamínica ${ }^{3}$} \\
\hline $\begin{array}{l}\text { Fibra (g) Goma guar } \\
\text { parcialmente hidrolisada }^{5}\end{array}$ & 4,30 & 10,00 \\
\hline Agua destilada (g) & & 767,60 \\
\hline Total $(\mathrm{g})$ & 100,00 & 1000,00 \\
\hline
\end{tabular}

${ }^{1}$ Samprosoy NB 90 (Bungue Alimentos do Brasil Ltda.); ${ }^{2}$ Mor-Rex ${ }^{\circledast}$ 1920 (Corn Products Brasil-Ingredientes Industriais Ltda.); ${ }^{3}$ Support Produtos Nutricionais Ltda.; ${ }^{4}$ Lecitina de soja (Bungue Alimentos do Brasil Ltda.); ${ }^{5}$ Novartis Consumer Health Ltda.; ${ }^{6} 1 \mathrm{~L}$ de dieta diluída apresenta 232,4 g de pó

Tabela 2. Composição da mistura salina da dieta experimental

\begin{tabular}{|c|c|c|c|}
\hline $\begin{array}{l}\text { Sais } \\
\text { Valor em } \\
100 \mathrm{~g} \text { da mistura } \\
\text { salina }\end{array}$ & Elemento & $\begin{array}{c}\text { Quantidade do } \\
\text { elemento em } \\
100 \mathrm{~g} \text { da mistura } \\
\text { salina }\end{array}$ & $\begin{array}{r}\text { Quantidade } \\
\text { do elemento } \\
\text { em } 1 \mathrm{~L} \mathrm{de} \\
\text { dieta diluída }\end{array}$ \\
\hline $\mathrm{FeSO}_{4} \cdot 7 \mathrm{H}_{2} 01,00 \mathrm{~g}$ & $\mathrm{Fe}$ & $200,00 \mathrm{mg}$ & $10,00 \mathrm{mg}$ \\
\hline $\mathrm{MgCO}_{3} 8,00 \mathrm{~g}$ & $\mathrm{Mg}$ & $2,29 \mathrm{~g}$ & $115,00 \mathrm{mg}$ \\
\hline \multirow[t]{2}{*}{$\mathrm{KH}_{2} \mathrm{PO}_{4}^{3} 48,00 \mathrm{~g}$} & $\mathrm{P}$ & $11,13 \mathrm{~g}$ & $557,00 \mathrm{mg}$ \\
\hline & $\mathrm{K}$ & $14,04 \mathrm{~g}$ & $702,00 \mathrm{mg}$ \\
\hline $\mathrm{ZnSO}_{4} \cdot 7 \mathrm{H}_{2} 00,316 \mathrm{~g}$ & $\mathrm{Zn}$ & $72,00 \mathrm{mg}$ & $3,60 \mathrm{mg}$ \\
\hline $\mathrm{KIO}_{3} 0,024 \mathrm{~g}$ & I & $14,40 \mathrm{mg}$ & $0,72 \mathrm{mg}$ \\
\hline $\mathrm{MnSO}_{4} \cdot \mathrm{H}_{2} 00,054 \mathrm{~g}$ & $\mathrm{Mn}$ & $17,64 \mathrm{mg}$ & $0,88 \mathrm{mg}$ \\
\hline $\mathrm{CuSO}_{4} \cdot 5 \mathrm{H}_{2}^{2} 00,046 \mathrm{~g}$ & $\mathrm{Cu}$ & $11,88 \mathrm{mg}$ & $0,59 \mathrm{mg}$ \\
\hline \multirow[t]{2}{*}{$\mathrm{NaCl} 24,00 \mathrm{~g}$} & $\mathrm{Na}$ & $9,22 \mathrm{~g}$ & $461,00 \mathrm{mg}$ \\
\hline & $\mathrm{Cl}$ & $14,24 \mathrm{~g}$ & $712,00 \mathrm{mg}$ \\
\hline
\end{tabular}

Maltodextrina $18,56 \mathrm{~g}$

Total $100 \mathrm{~g}$

${ }^{1}$ Diluição: $5 \mathrm{~g}$ de mistura salina/1 L de dieta. Todos os sais utilizados no preparo da mistura salina continham a informação de grau P.A. dos respectivos fabricantes

Método para determinação do ferro e do zinco dialisado

Para determinação do ferro e do zinco dialisado foi utilizado o método in vitro proposto por Miller et al..$^{13} \mathrm{e}$ modificado por Luten et al. ${ }^{14}$ para avaliação da biodisponibilidade de minerais em alimentos e dietas, que envolve a simulação da digestão gastrintestinal, seguida da determinação do mineral solúvel e que consiste de duas etapas básicas que simulam a digestão gástrica e duodenal.

Em linhas gerais, a dieta enteral foi submetida à digestão com pepsina, após acidificação do meio com $\mathrm{HCl} 6 \mathrm{~N}$ até $\mathrm{pH} 2$, seguida de digestão com pancreatina/bile, após a alcalinização do meio a pH 7, com $\mathrm{NaHCO}_{3}$ contido em tubos de diálise.

No final do último período de digestão, os segmentos de tubos de diálise foram lavados com água desionizada e seu conteúdo colocado em balão volumétrico de $25 \mathrm{~mL}$, sendo o volume final completado com água desionizada, condicionado em freezer até o momento da leitura.

\section{Método para determinação do ferro e do zinco total}

Para a determinação da concentração de ferro e zinco total presente nas dietas enterais foi utilizado o método de espectrometria de absorção atômica (EAA), segundo as normas da AOAC. As dietas enterais foram digeridas com ácido nítrico $\left(\mathrm{HNO}_{3}\right)$ e peróxido de hidrogênio $\left(\mathrm{H}_{2} \mathrm{O}_{2}\right)$ em proporção de $5: 1$, a $100{ }^{\circ} \mathrm{C}$, em bloco digestor $\left(\right.$ Pyrotec $\left.^{\circledR}\right)$, sendo diluídas com água deionizada em um balão de $50 \mathrm{~mL}$.

As leituras das amostras e das curvas padrões foram realizadas no Polarized Zeeman AAS Hitachi Z-5000, por chama e ar/acetileno oxidante, nas seguintes condições: lâmpada de cátodo oco, comprimento de onda de $248,3 \mathrm{~nm}$ para o ferro e de $213,9 \mathrm{~nm}$ para o zinco; fenda de $0,2 \mathrm{~nm}$ para o ferro e o zinco. As soluções da curva padrão do ferro foi o Cloreto Férrico Titrisol Merck ${ }^{\circledR}$ 9972, nas concentrações de 0,$1 ; 0,2 ; 0.3 ; 0,5 ; 1,0 ; 3,0$ e $5,0 \mu \mathrm{g} \mathrm{Fe} / \mathrm{mL}$ e as de zinco a partir da solução de Cloreto de Zinco Titrisol Merck ${ }^{\circledR}$ 9953, nas concentrações de 0,$1 ; 0,2 ; 0,3 ; 0,5 ; 1,0 ; 3,0$ e $5,0 \mu \mathrm{g} \mathrm{Zn} / \mathrm{mL}$.

\section{Metodologia de superfície de resposta para misturas: Delineamento experimental}

As variáveis independentes selecionadas foram TCM $\left(\mathrm{x}_{1}\right)$, Fibra $\left(\mathrm{x}_{2}\right)$ e Cálcio $\left(\mathrm{x}_{3}\right)$. Tratando-se de uma formulação em pó para dieta enteral, as variáveis devem obedecer à relação

$$
\sum_{i=1}^{q} \mathrm{X}_{\mathrm{i}}=1,0=100 \%
$$

A equação polinomial, que descreve o modelo mais simples (linear) para os três componentes da mistura de interesse para se avaliar a disponibilidade de magnésio dialisado, pode ser representada por: $y_{i}=\beta_{0}+\beta_{1} x_{1}+\beta_{2} x_{2}+\beta_{3} x_{3}+\varepsilon$, onde $y_{i}$ é o valor da propriedade de interesse, $\beta_{0}$ e $\beta_{i}^{\text {ss }}$ são os coeficientes do modelo a serem estimados pelo método dos mínimos quadrados, $x_{i}$ representa as variáveis dependentes codificadas e $\varepsilon$ é o erro aleatório. ${ }^{15,16}$

Multiplicando-se $\beta_{0}$ pela identidade $\left(\mathrm{x}_{1}+\mathrm{x}_{2}+\mathrm{x}_{3}\right)$ e isolando-se as variáveis, tem-se a chamada equação polinomial canônica de Sheffé ou polinomial $\{\mathrm{q}, \mathrm{m}\}$ onde q é igual ao número de componentes e $m$ ao grau da equação. No caso de linearidade $\{3,1\}$ teremos: $\mathrm{y}_{\mathrm{i}}=\mathrm{b}^{*}{ }_{1} \mathrm{x}_{1}+\mathrm{b}^{*}{ }_{2} \mathrm{x}_{2}+\mathrm{b}^{*}{ }_{3} \mathrm{x}_{3}$, onde $\mathrm{b}^{*}{ }_{\mathrm{i}}=\mathrm{b}_{0}+\mathrm{b}_{\mathrm{i}}$ e teremos ainda: ${ }^{11}$

$\mathrm{y}_{\mathrm{i}}=\mathrm{b}{ }_{1} \mathrm{x}_{1}+\mathrm{b}_{2} \mathrm{x}_{2}+\mathrm{b}_{3} \mathrm{x}_{3}$ (modelo linear) onde $b^{*}{ }_{i}=b_{0}+b_{i}$

$$
\begin{aligned}
& \begin{array}{l}
\mathrm{y}_{\mathrm{i}}=\mathrm{b}^{*}{ }_{1} \mathrm{x}_{1}+\mathrm{b}^{*} \mathrm{x}_{2}+\mathrm{b}^{*} \mathrm{x}_{3}+\mathrm{b}^{*}{ }_{12} \mathrm{x}_{1} \mathrm{x}_{2}+\mathrm{b}^{*}{ }_{13} \mathrm{x}_{1} \mathrm{x}_{3}+\mathrm{b}^{*}{ }_{23} \mathrm{x}_{2} \mathrm{x}_{3} \\
\text { onde } \mathrm{b}^{*}{ }_{\mathrm{i}}=\mathrm{b}_{0}+\mathrm{b}_{\mathrm{i}}+\mathrm{b}_{\mathrm{ii}}
\end{array} \\
& \begin{array}{l}
\text { (modelo quadrático) } \\
\mathrm{y}_{\mathrm{i}}=\mathrm{b}^{*}{ }_{1} \mathrm{x}_{1}+\mathrm{b}^{*}{ }_{2} \mathrm{x}_{2}+\mathrm{b}^{*}{ }_{3} \mathrm{x}_{3}+\mathrm{b}^{*}{ }_{12} \mathrm{x}_{1} \mathrm{x}_{2}+\mathrm{b}^{*}{ }_{13} \mathrm{x}_{1} \mathrm{x}_{3}+\mathrm{b}^{*}{ }_{23} \mathrm{x}_{2} \mathrm{x}_{3}+\mathrm{b}^{*}{ }_{123} \mathrm{x}_{1} \mathrm{x}_{2} \mathrm{x}_{3} \\
\text { (modelo cúbico especial) }
\end{array}
\end{aligned}
$$

Portanto, para a estimativa do valor dos coeficientes $b^{*}{ }_{i}$ são necessários pelo menos 3 ensaios experimentais. Da mesma forma, para modelos mais complexos, como o quadrático ou o cúbico especial, necessita-se de 6 e 7 ensaios, respectivamente. Como a diferença nos termos do delineamento entre o modelo quadrático e o modelo cúbico especial é de apenas um ensaio experimental, ${ }^{15}$ optou-se pela utilização de um planejamento experimental do tipo centróide simplex com 7 ensaios experimentais, buscando posteri- 
Tabela 3. Formulações das dietas experimentais que compuseram o delineamento experimental

\begin{tabular}{lccccccc}
\hline Ingredientes $(\mathrm{g})$ & Dieta 1 & Dieta 2 & Dieta 3 & Dieta 4 & Dieta 5 & Dieta 6 & Dieta 7 \\
\hline Isolado Protéico de Soja & 31,0 & 31,0 & 31,0 & 31,0 & 31,0 & 31,0 & 31,0 \\
Maltodextrina & 148,4 & 131,4 & 131,4 & 139,9 & 131,4 & 139,9 & 137,0 \\
Óleo de Milho & - & 17,0 & 17,0 & 8,5 & 17,0 & 8,5 & 11,3 \\
Óleo de Canola & 18,0 & 18,0 & 18,0 & 18,0 & 18,0 & 18,0 & 18,0 \\
TCM & 17 & - & - & 8,5 & - & 8,5 & 5,7 \\
Lecitina de Soja & 3,0 & 3,0 & 3,0 & 3,0 & 3,0 & 3,0 & 3,0 \\
Fibra & - & 17 & - & 8,5 & 8,5 & 5,0 & 5,7 \\
Mistura Salina & 5,0 & 5,0 & 5,0 & 5,0 & 5,0 & 8,0 & 5,0 \\
Carbonato de Cálcio & - & - & 17 & - & 8,5 & 10,0 & 10,0 \\
Mistura Vitamínica & 10,0 & 10,0 & 10,0 & 10,0 & 10,0 & 767,6 & 767,6 \\
Água & 767,6 & 767,6 & 767,6 & 767,6 & 767,6 & 100000 & 1000,0 \\
Total (g) & 1000,0 & 1000,0 & 1000,0 & 1000,0 & 1000,0 & 1000,0 \\
\hline
\end{tabular}

${ }^{1}$ Mistura de carbonato de cálcio contendo 15,0 g de maltodextrina e 2,0 g de carbonato de cálcio

ormente sua simplificação através de testes de significância de seus coeficientes $^{15-17}$ (Figura 1).

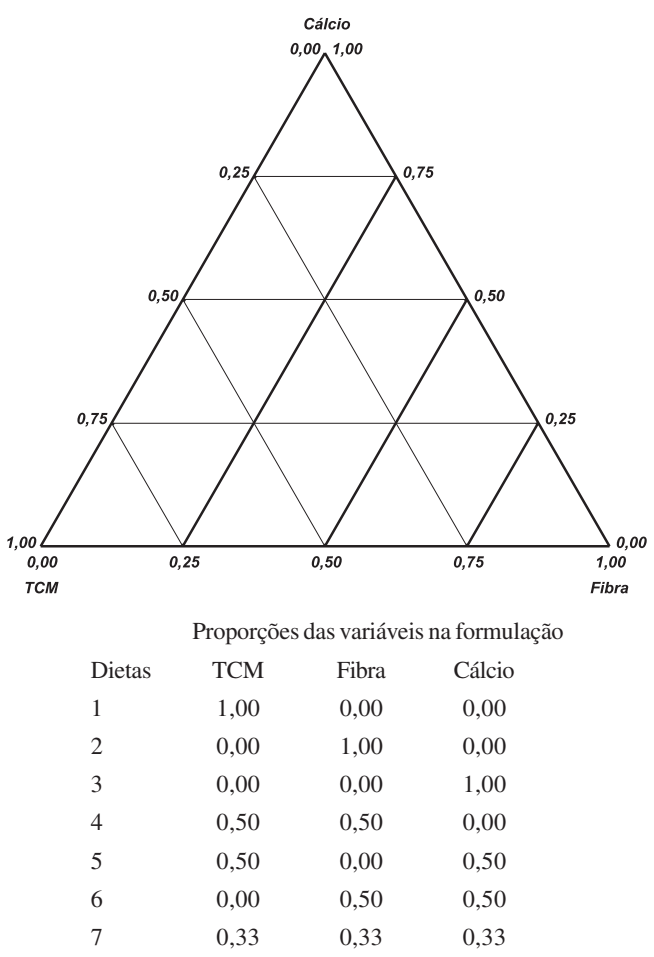

Figura 1. Delineamento "centróide-simplex"

\section{Análise estatística}

A estimativa do valor dos coeficientes de todas as regressões foi obtida pelo método dos mínimos quadrados, sendo sua significância avaliada pelo teste t, adotando-se um valor de $\mathrm{p} \leq$ 0,05 , para todos os ensaios.

A adequação dos modelos propostos foi avaliada pela análise do resíduo, classificado como "falta de ajuste" e "erro puro", comparando-se a proporção da variação que é explicada pelo modelo, isto é, pela análise do coeficiente de determinação $\mathrm{R}^{2}$.

Uma vez obtido um modelo polinomial ajustado à resposta, sua otimização foi feita pela técnica proposta para variáveis dependentes. ${ }^{18}$ Esta baseia-se na definição de uma função de desejabilidade restrita no intervalo de [0,1], para a qual se adotou como limites inferior, médio e superior nos valores de 0; 0,5 e 1,0, respectivamente. Os cálculos e gráficos foram elaborados utilizan- do-se o programa Statistica V. $6{ }^{19}$

\section{RESULTADOS}

Para os minerais avaliados (ferro e zinco) foram obtidos os modelos matemáticos pela técnica estatística de superfície de resposta para misturas. As porcentagens de dialisabilidade para ferro e zinco obtidas pelas condições estabelecidas no delineamento experimental estão representadas na Tabela 4 .

Para as respostas porcentagem de ferro e zinco dialisados, a Tabela 5 apresenta os fatores de variação obtidos pela análise de variância para cada um dos modelos empíricos. Pode-se observar os valores de distribuição do teste $\mathrm{F}$, do nível de significância estatística $\mathrm{p}$ e do coeficiente de determinação $\mathrm{R}^{2}$ para se verificar a adequação dos modelos às respostas avaliadas para cada um dos minerais.

Três repetições foram preparadas para cada ensaio e os valores obtidos para a estimativa da resposta $\hat{y}$ "porcentagem de ferro dialisado" foram utilizados para a obtenção de um modelo quadrático ajustado pelos dados experimentais, para predizer a relação da resposta com os três nutrientes do delineamento experimental, conforme mostra a Equação 1:

$$
\hat{y}=\underset{(0,31) \quad(0,31) \quad(0,34) \quad(1,57) \quad+3 x_{1}+4,50 x_{2}+1,30 x_{3}+5,32 x_{1} x_{3}+15,42 x_{2} x_{3}}{(1,57)}
$$

A Equação 1 apresenta os coeficientes de regressão do modelo quadrático ajustado pelos dados experimentais para o ferro e seus respectivos erros padrões, estimados pelos dados experimentais no modelo empírico.

A Figura 1S Material Suplementar apresenta as curvas de contorno obtidas para a resposta $\hat{y}(\mathrm{x})$ porcentagem de ferro dialisado para as 3 variáveis $\left(\mathrm{x}_{1}, \mathrm{x}_{2}, \mathrm{x}_{3}\right)$, nas quais se observa que os maiores valores de $\hat{\mathrm{y}}(\mathrm{x})$ estão associados à interação fibra e cálcio. A Figura $2 \mathrm{~S}$ mostra a análise de variância que foi realizada com os dados experimentais obtidos através da reprodução na dieta enteral nas proporções apresentadas na Figura 1S. Pode-se concluir que os resultados foram validados.

Os resultados obtidos para a dialisabilidade de zinco a resposta $\hat{y}$ "porcentagem de zinco dialisado" foram caracterizados em um modelo quadrático ajustado pelos dados experimentais, mostrado na Equação 2, no qual podem ser observados os valores dos coeficientes de regressão e o erro padrão para cada um dos nutrientes e para as interações binárias.

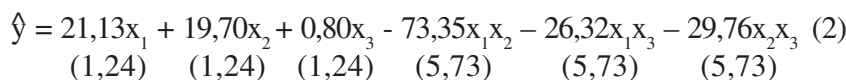


Tabela 4. Porcentagem de ferro $(\% \mathrm{FeD})$ e zinco $(\% \mathrm{ZnD})$ dialisados com diferentes proporções de TCM, fibra e cálcio na formulação

\begin{tabular}{lccccc}
\hline Dietas & TCM & Fibra & Cálcio & Média $(\%$ FeD $)$ & Média $(\% \mathrm{Zn})$ \\
\hline 1 & 1 & 0 & 0 & $5,40(0,44)$ & $21,30(3,50)$ \\
2 & 0 & 1 & 0 & $4,32(0,10)$ & $19,88(3,80)$ \\
3 & 0 & 0 & 1 & $1,25(0,18)$ & $1,02(0,12)$ \\
4 & 0,5 & 0,5 & 0 & $5,50(0,49)$ & $3,70(1,65)$ \\
5 & 0 & 0,5 & 0,5 & $6,90(0,64)$ & $1,40(0,30)$ \\
6 & 0,5 & 0 & 0,5 & $4,90(0,64)$ & $2,13(0,50)$ \\
7 & 0,33 & 0,33 & 0,33 & $5,70(0,33)$ & $1,02(0,07)$ \\
\hline
\end{tabular}

$(n=3)$

Tabela 5. Fatores de variação para as respostas de porcentagem de ferro e zinco dialisados

\begin{tabular}{lccc}
\hline Nutrientes & $\begin{array}{c}\text { Valor de } \\
\mathrm{F}\end{array}$ & $\begin{array}{c}\text { Valor de } \\
\mathrm{p}^{* *}\end{array}$ & $\begin{array}{c}\text { Coeficiente de } \\
\text { Determinação }\left(\mathrm{R}^{2}\right)\end{array}$ \\
\hline Ferro & 39,08 & 0,0000 & 0,89 \\
Zinco & 64,34 & 0,0000 & 0,94 \\
\hline
\end{tabular}

(**) significativo a nível de $95 \%$ de probabilidade $(\mathrm{p}<0,01)$

Esses coeficientes apresentaram valores de $\mathrm{b}_{1}^{*} \mathrm{x}_{1}, \mathrm{~b}_{2}{ }_{2} \mathrm{x}_{2}, \mathrm{~b}_{3} \mathrm{x}_{3}$ $\mathrm{e} \mathrm{b}^{*}{ }_{12} \mathrm{x}_{1} \mathrm{x}_{2}, \mathrm{~b}^{*}{ }_{13} \mathrm{x}_{1} \mathrm{x}_{3}$ e b ${ }_{23} \mathrm{x}_{2} \mathrm{x}_{3}$ onde $\mathrm{b}^{*}{ }_{12}$ e b ${ }^{*}{ }_{13}$ e b ${ }^{*}<0$, o que significou um antagonismo entre as interações binárias avaliadas para a resposta.

A Figura $3 \mathrm{~S}$ apresenta as curvas de contorno obtido para a resposta $\hat{\mathrm{y}}(\mathrm{x})$ porcentagem de zinco dialisado para as 3 variáveis $\left(\mathrm{x}_{1}\right.$, $\left.\mathrm{x}_{2}, \mathrm{x}_{3}\right)$, na qual se observa que os maiores valores de $\hat{y}(\mathrm{x})$ estão associados aos triacilglicerídeos de cadeia média e à fibra, porém, todas as interações binárias foram negativas de acordo com os coeficientes mostrados.

As Figuras $4 \mathrm{~S}$ e $5 \mathrm{~S}$ mostram o resultado da formulação otimizada para ferro e zinco, respectivamente, assim como os valores de desejabilidade da porcentagem de dialisabilidade para os dois minerais em função de cada variável estudada.

\section{DISCUSSÃO}

Todos os modelos de regressão (linear, quadrático e cúbico especial) para os valores de ferro e zinco dialisados se mostraram altamente significativos $(\mathrm{p}<0,05)$. Portanto, para todos os modelos rejeitamos a hipótese de nulidade $\left(H_{0}=\beta_{1}=\beta_{2}=\beta_{3}\right)$, demonstrando a dependência das respostas nas proporções dos nutrientes estudados nas misturas elaboradas no delineamento. Para a verificação da adequação dos modelos globais, foi determinante o valor de $\mathrm{F}$ calculado maior que o $F$ tabelado $\left(\mathrm{F}_{4,16}=3,01\right.$ para ferro e $\mathrm{F}_{5,15}=2,90$ para zinco), e não houve evidência de falta de ajuste $\left(\mathrm{F}_{2,14}=3,74\right.$ para ferro e $F_{1,14}=4,60$ para zinco) para um nível de $95 \%$ de significância. ${ }^{15}$

A técnica empregada possibilita a otimização de processos de produção e industriais, através do qual se permite avaliar os efeitos das interações entre as variáveis e as respostas, salientando sua aplicabilidade na indústria de alimentos e capacidade de potencialização das propriedades funcionais dos alimentos. ${ }^{20}$

Dentre todos os efeitos sinérgicos observados para o ferro dialisado, o efeito mais pronunciado foi da interação binária fibra e cálcio. Por serem altamente fermentáveis, atuarem através da ação bacteriana no cólon e apresentarem uma capacidade de ligação com minerais, principalmente com o cálcio, as fibras solúveis têm sido recomendadas em dietas enterais. A capacidade de translocação dos locais absortivos do cálcio do intestino delgado para o ceco e cólon, onde, ao serem degradadas, aumentam a produção de ácidos graxos de cadeia curta, leva a uma diminuição do pH, o que induziria o aumento da absorção do cálcio nessa região do intestino. ${ }^{21}$

A goma guar e a goma guar parcialmente hidrolisada são mais importantes que outros tipos de fibras na produção de ácidos graxos de cadeia curta, por atuarem na microflora intestinal em humanos. ${ }^{21,22}$ Spacen et al. ${ }^{23}$ observaram que pacientes com íleo paralítico mostraram menor incidência de diarréia e menor comprometimento das funções intestinais, quandosubmetidos à nutrição enteral com fibra solúvel. Nesse aspecto, a goma guar hidrolisada foi mais efetiva que outros tipos de fibra, devido às suas características para maior produção de ácidos graxos de cadeia curta no cólon.

Torre et $a l .{ }^{24}$, ao estudarem as interações de $\mathrm{Fe}^{2+}, \mathrm{Ca}^{2+}$ e $\mathrm{Fe}^{3+}$ em dietas enterais através de métodos in vitro simulando condições digestivas em diferentes concentrações de fibra solúvel e insolúvel nas dietas e diferentes $\mathrm{pHs}$, observaram que altas quantidades de fibra e condições físico-químicas não adequadas podem levar a uma má absorção de cátions no organismo humano. A dialisabilidade de cálcio foi de 2,5\% em média e sofreu interferências devido à presença de maiores quantidades de fibra nas dietas, mostrando que os componentes da fibra e os íons metálicos cálcio e ferro podem ser muito afetados com esses nutrientes.

Torre $e t a l .^{25}$ estudaram os efeitos das fibras alimentares utilizando frações isoladas de fibra, para avaliar a disponibilidade de ferro, cálcio e zinco, por métodos in vitro e in vivo, e observaram que em humanos a goma guar tem a capacidade de se agrupar em estruturas onde o mineral passa a fazer parte da composição estrutural e química do composto sem formar colóides e complexos de difícil hidrólise, mecanismo observado tanto para $\mathrm{Fe}^{2+}$ como para $\mathrm{Zn}^{2+}$.

Gupta et al..$^{26}$ ao avaliarem a biodisponibilidade de cálcio e ferro em vegetais folhosos, pelo método in vitro de diálise, concluíram que os componentes presentes na estrutura química desses alimentos, como oxalatos, fibras, taninos e acido fítico, são os principais interferentes da biodisponibilidade de ferro.

Oliveira e Osório ${ }^{27}$ salientaram que o consumo de leite de vaca na primeira infância pode aumentar a incidência de anemia ferropriva em crianças, porque o alimento apresenta baixa biodisponibilidade e densidade para o ferro, excesso de proteínas e cálcio, além de poder interferir na absorção do ferro proveniente de outros alimentos na dieta.

Perales et ll $^{28}$ ao avaliarem o efeito da biodisponibilidade do cálcio, ferro e zinco em amostras fortificadas ou não com cálcio, de leite de vaca, através dos métodos in vitro do dialisado e do cultivo celular Caco 2, mostraram que a própria matriz tende a diminuir a biodisponibilidade do cálcio, verificado nos leites não fortificados, o que pode ser explicado pela interação do cálcio com os componentes do leite, principalmente com a proteína do leite, e formação de 
compostos insolúveis que tendem a prejudicar o aproveitamento do mineral. O leite fortificado diminui a biodisponibilidade de ferro e zinco, pela interação desses nutrientes com o cálcio, e os autores concluíram que a interação entre os minerais e o leite desfavorece que o alimento seja utilizado em programas de combate a carências nutricionais de minerais.

Na mistura avaliada, a interação binária TCM-cálcio foi outro fator importante para a dialisabilidade do ferro. Essa interação pode estar indicando um mecanismo semelhante ao descrito, por diversos autores para a interferência entre fibra solúvel e cálcio. ${ }^{28}$ Assim, alimentos ou dietas que contenham compostos de estruturas menos complexas (como os TCMs) podem ligar o cálcio, em presença de grandes quantidades do mineral e com isso favorecer a disponibilidade do ferro nas dietas enterais.

Rodrigues et $_{\text {al }}{ }^{29}$ mostraram que a gordura presente no leite, caracterizada em fontes naturais dessa matriz, se constitui de quantidades razoáveis de colesterol e ácidos graxos saturados, como os triacilgliceróis de cadeia média. Toba et al. ${ }^{30}$ ao compararem os efeitos dos componentes do leite na biodisponibilidade do cálcio, nos ratos em crescimento, concluíram que o mineral apresentou interações com os componentes do leite, mostrando a interferência da própria estrutura química do leite na absorção do cálcio, devido à formação de compostos insolúveis tender a diminuir a disponibilidade do mineral.

Yang et al. ${ }^{31} \mathrm{em}$ um estudo de metanálise, em que foi avaliada a utilização das fibras em fórmulas de alimentação enteral, mostraram que houve redução do tempo de internação hospitalar de pacientes transplantados de fígado e de pacientes com intervenção cirúrgica abdominal. Para os casos de diarréia e infecção, em que foi empregada a fibra na alimentação, foi observado um controle nos pacientes transplantados de fígado, de intervenção cirúrgica abdominal e com íleo paralítico, em razão de um benefício no quadro clínico dos pacientes.

A forma química dos minerais e fibras contidas em alimentos ou em dietas, principalmente na presença de oxalatos e fitatos, impede a absorção de ferro, zinco, cobre e cálcio. Por isso, em dietas enterais, existe a preocupação de serem adicionados substratos que não venham a impedir a biodisponibilidade de nutrientes, levando pacientes à desnutrição de minerais essenciais para as funções metabólicas do organismo. ${ }^{24,25,28}$

Em nossos estudos, de acordo com os resultados obtidos, todas as interações estudadas se mostraram estatisticamente significativas para a disponibilidade de zinco, porém indicaram o antagonismo da resposta em relação ao ferro. De acordo com Solomons e Ruiz ${ }^{32}$ existe uma interação antagônica entre zinco e ferro presentes em dietas e alimentos. Uma vez que esses íons são quimicamente semelhantes e quando juntos no lúmen intestinal competem pelos mesmos sítios de absorção, e pelo zinco apresentar maior massa molecular que o ferro $(\mathrm{MMZn}=65,37 / \mathrm{MMFe}=55,85)$, este tende a excluir o ferro no momento da entrada do íon na célula intestinal, deixando de ser absorvido pela borda em escova no enterócito. Dois mecanismos podem explicar sua variabilidade biológica: o de competição e o de co-adaptação. A principal diferença entre eles está na quantidade de ferro e zinco contidos em alimentos ou dietas alimentares. A característica necessária para que ocorra o processo de adaptação é manter as quantidades de ferro e zinco iguais em $\mathrm{mg} / \mathrm{L}$ no caso das dietas enterais. Para que ocorra o processo de competição pequenas quantidades de ferro em relação ao zinco (2:1, respectivamente) passam a caracterizar um processo de competição e um antagonismo entre os dois minerais fica evidente.

Em uma avaliação para o zinco dialisado na formulação, podese observar pelos dados experimentais que as variáveis selecionadas, para a aplicação do delineamento experimental, não foram ade- quadas. Isso pode ser mostrado pelos contornos das curvas de nível no qual a resposta não definiu uma região de maximização. Isso também pode ser observado pelo antagonismo das interações binárias que foram significativas mas mostraram que as variáveis selecio-nadas foram prejudiciais para a disponibilidade do mineral na formulação.

Ao verificarmos a otimização da formulação pode-se concluir que apenas uma das variáveis, o TCM, foi responsável pela maximização da resposta, confirmando que as variáveis TCM e fibra se mostraram competitivas entre elas na formulação, não sendo possível desenhar uma otimização que mostrasse um ajuste na proporção das mesmas na formulação.

Os TCMs quando ministrados em dietas enterais, para pacientes no período pós-operatório, reduzem significativamente o risco de complicações no trato gastrintestinal e de infecções, por modularem a produção de eicosanóides antiinflamatórios, e riscos de problemas renais e hepáticos por auxiliarem no metabolismo de lipídeos. ${ }^{33}$ Porém, para o estudo foi a variável que mostrou mais claramente que ao otimizar o zinco na formulação prejudicou-se o ferro, resultados que podem ser observados nas Figuras $4 \mathrm{~S}$ e $5 \mathrm{~S}$.

Perales et al..$^{34}$ ao avaliarem a biodisponibilidade de zinco em fórmulas infantis, pelo método in vitro de diálise, concluíram que o zinco sofre interferências pelos componentes desses alimentos, diminuindo a sua biodisponibilidade. Os efeitos de interação entre os nutrientes puderam ser observados através da aplicabilidade de um método que simula as condições digestivas do trato gastrintestinal humano, através do qual se detectou a formação de complexos insolúveis com as estruturas de baixo peso molecular, entre elas, aminoácidos e cálcio, provenientes da fonte proteíca. Os autores ressaltaram que os resultados podem ser correlacionados com os obtidos com humanos quando se visa avaliar o aproveitamento de nutrientes provenientes nas fórmulas infantis.

Ma et al..$^{35}$ ao avaliarem a dieta chinesa, rica em cereais e leguminosas, como trigo, arroz, milho e soja, observaram que esses alimentos apresentaram quantidades diferentes de fitatos, devido às diferentes espécies, e que houve uma diminuição estatisticamente significativa na biodisponibilidade de cálcio, ferro e zinco inversamente proporcional às quantidades de fitato. Observouse que o fato pode ser explicado em razão de uma propriedade ligante do fitato com os minerais, presentes nos alimentos, o que levou à formação de compostos insolúveis que impedem o aproveitamento dos nutrientes. Os autores ressaltaram a importância de estudos de interações entre os nutrientes e de processos de otimização para minimizar esses efeitos, principalmente, em populações que apresentam hábitos alimentares específicos.

Porres et al. ${ }^{36}$ ao estudarem o efeito da fermentação natural de cereais e leguminosas no trato gastrintestinal em relação ao aproveitamento de minerais, optaram por utilizar o método in vitro de diálise, por simulação das condições do sistema digestório humano. Mostraram que foi possível a caracterização da composição química dos alimentos nas etapas de digestão e absorção, em decorrência das condições metodológicas pré-estabelecidas, e concluíram que diferentes condições fermentativas associadas as condições de digestão/absorção mostraram maior ou menor caracterização da interação entre ferro e zinco, em um mecanismo de competição, intensificado na fase absortiva, onde foram verificadas concentrações inversamente proporcionais entre os dois minerais.

O emprego da modelagem matemática e de processos de otimização de formulações alimentícias pode ser um importante diferencial para a avaliação da qualidade nutricional de alimentos e dietas para diversos fins. A facilidade de avaliação multifatorial, usando técnicas de modelagem matemática, amplia a gama de conhecimentos para o pesquisador porque fornece ferramentas capa- 
zes de correlacionar múltiplos fatores, que no caso da nutrição e alimentação inclui os hábitos alimentares dos indivíduos, as quantidades de nutrientes presentes nas dietas e alimentos, fatores econômicos e culturais das populações de estudo, agricultura local e favorecimento climático para a oferta de alimentos, entre outros. ${ }^{37}$

\section{CONCLUSÃO}

Verificou-se um antagonismo entre os dois minerais e a necessidade de se mensurar o efeito dos dois minerais em formulações de alimentos e de dietas quando se visa a maximização do ferro e do zinco.

\section{MATERIAL SUPLEMENTAR}

No material suplementar disponível em http://quimicanova. sbq. org. br, na forma de arquivo PDF, com acesso livre, encontram-se as Figuras $1 \mathrm{~S}$ a $5 \mathrm{~S}$

\section{AGRADECIMENTOS}

À Faculdade de Ciências Farmacêuticas da Universidade de São Paulo, ao Departamento de Alimentos e Nutrição Experimental, ao Laboratório de Minerais, pela possibilidade de realização deste trabalho.

\section{REFERÊNCIAS}

1. Harvey, L.; J. Nutr. 2001, 31, 179

2. Reddy, M. B.; Hurrel, R. F.; Cook, J. D.; J. Nutr. 2006, 136, 576.

3. Zimmermann, M. B.; Biebinger, R.; Rohner, F.; Am J Clin Nutr. 2006, 84. 580 .

4. Winichagoon, P.; Mckenzie, J. E.; Chavasit, V.; Pongcharoen, T.; Gowachirapant, S.; Boonpraderm, A.; J. Nutr. 2006, 136, 1617.

5. Proulx, A. K.; Reddy, M. B.; J. Agric. Food Chem. 2006, 54, 518

6. Pallarés, I. F.; Aliaga, I. L.; Barrionuevo, M.; Alférez, M. J.; Campos, M. S.; Br. J. Nutr. 1993, 70, 609.

7. Narasinga-Rao, B. S.; J. Food Sci. Technol. 1994, 31, 353.

8. Pushpanjali, K.; Khokhar, S.; Food Chem. 1996, 56, 111.
9. Chiplonkar, S. A.; Agte, V. V.; Tarwadi, K. V.; Kavadia, R.; Biol. Trace Elem. Res. 1999, 67, 249.

10. Kahyaoglu, T.; Kaya, S.; J. Sci. Food Agric. 2006, 86, 1452.

11. Association of Official Analytical Chemistrs, AOAC; Official Methods of Analysis, AOAC: Washington 1995.

12. Reeves, P. G.; Nielsen, F. H.; Fahey J. R. G. C.; J. Nutr. 1993,123, 1939.

13. Miller, D. D.; Schricker, B. R.; Rasmussen, R. R.; Van Campen D.; Am. J. Clin. Nutr. 1981, 34, 2248.

14. Luten, J.; Crews, H.; Flynn, A.; van Dael, P.; Kastenmayer, P.; Hurrel, R.; Deelstra, H.; J. Sci. Food Agric. 1996, 72, 415.

15. Barros Netos, B.; Scarminio, I. S.; Bruns, R. E.; Como Fazer Experimentos - Pesquisa e desenvolvimento na ciência e na indústria. $2^{\mathrm{a}}$ ed., Ed da UNICAMP: Campinas, 2001.

16. Cornell, J. A.; Experiments with mixtures, designs, models and the analysis of mixture data, $2^{\text {nd }}$. ed., John Wiley \& Sons: Nova YorK, 1990.

17. Castro, I. A.; Tirapegui, J.; Silva, R. S. S. F.; Nutr Res. 2000, 20, 1341.

18. Derringer, G.; Suich, R.; J. Qual. Technol. 1980, 12, 214.

19. Statistica; Data Analysis Software System; Tulsa, USA, 1998

20. Villarroel, M.; Pinno, L.; HAzbun, J.; Arch. Latinoam. Nutr., 2006, 56, 2.

21. Slavin, J. L.; Greenberg, N. A.; J. Nutr., 2003, 19, 549.

22. Velázquez, M.; Davies, C.; Marett, R.; Slavin, J. L.; Feirtag, J. M.; Anaerobe 2001, 6, 87 .

23. Spapen, H.; van Malderen, D. C.; Suys, O. E.; Huyghens, L.; Clin. Nutr. 2001, 20, 301 .

24. Torre, M.; Rodriguez, A. R.; Saura-Calixto, F.; Food Chem. 1995, 54, 23.

25. Torre, M.; Rodriguez, A. R.; Saura-Calixto, F.; Crit. Rev. Food Sci. Nutr. 1991, 30, 1 .

26. Gupta, S.; Lakshmi, A.; Prakash, B.; J. Sci. Food Agric. 2006, 86, 2147.

27. Oliveira, M. A. A.; Osório, M. M.; J. Pediatr. 2005, 81, 361.

28. Perales, S.; Barbera, R.; Lagarda, M. J.; Farre, R.; J. Agric. Food Chem. 2006, 54, 4901.

29. Rodrigues, J. N.; Gioielli, L. A.; Anton, C.; Cienc. Tecnol. Aliment. 2003 23,226

30. Toba, Y.; Tanaka, Y.; Tanaka, M.; Aoe, S.; Nutr. Res. 1999, 19, 449.

31. Yang, G.; Wu, X.; Zhou, Y.; Wang, Y.; World J. Gastroenterol. 2005, 11, 3935 .

32. Solomons, N. W.; Ruz, M.; Nutr. Res. 1997, 17, 177.

33. Tso, P.; Lee, T.; Demichele, S. J.; Am. Physiol. Soc. 1999, 333.

34. Perales, S.; Barbera, R.; Lagarda, M. J.; Farre, R.; J. Agric. Food Chem. 2006, 86,971 .

35. Ma, G.; Jin, Y.; Plao, J.; Kok, F.; Guusje, B.; Jacobsen, E.; J. Agric. Food Chem. 2005, 53, 10285.

36. Porres, J. M.; Aranda, P.; Lopez-Jurado, M.; Urbano, G.; J. Agric. Food Chem. 2003, 51, 5144.

37. Ferguson, E. L.; Darmon, N.; Fahmida, U.; Fitriyanti, S.; Harper, T. B.; Premachandra, I. M.; J. Nutr. 2006, 136, 2399. 


\section{Luciana Bueno}

Departamento de Alimentos e Nutrição Experimental, Divisão de Ciência dos Alimentos, Faculdade de Ciências Farmacêuticas, Universidade de São Paulo, Av. Lineu Prestes, 580, 05508-900 São Paulo - SP, Brasil
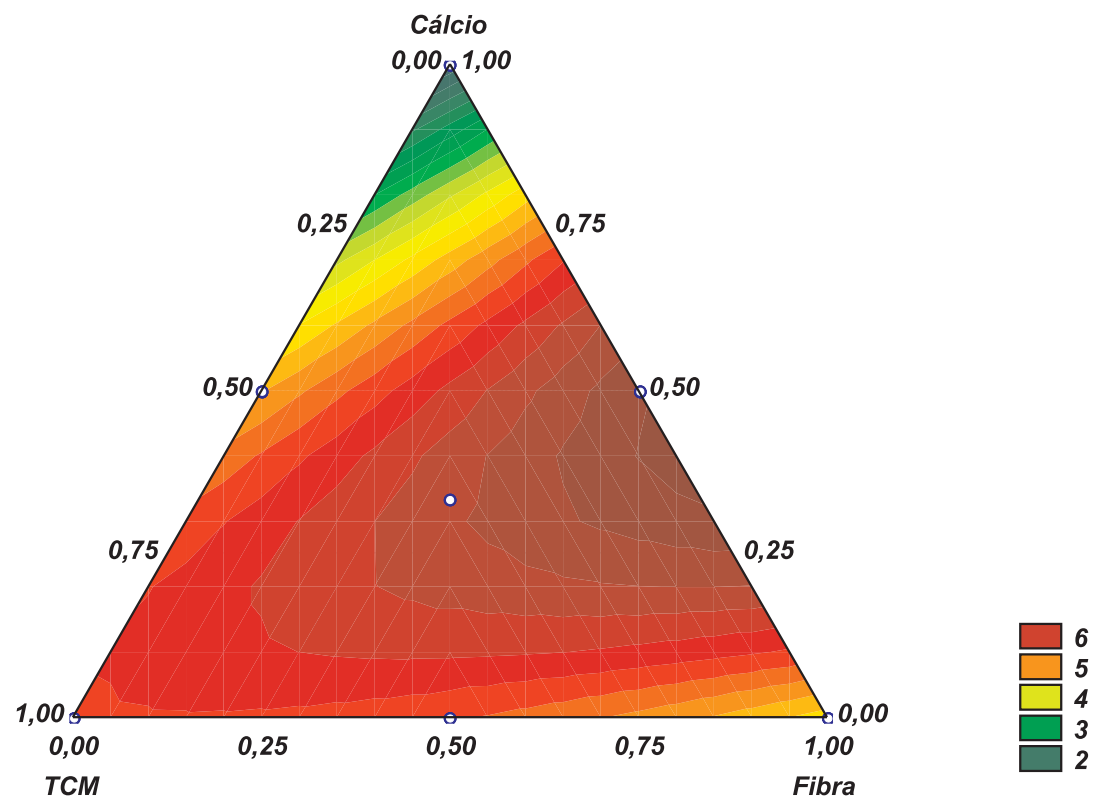

Figura 1S. Curvas de nível para a resposta do ferro dialisado

Variação entre a \% de ferro dialisado dos dados experimentais e a \% de ferro obtida pela formulação otimizada

\%ferro dialisado vs. valor otimizado

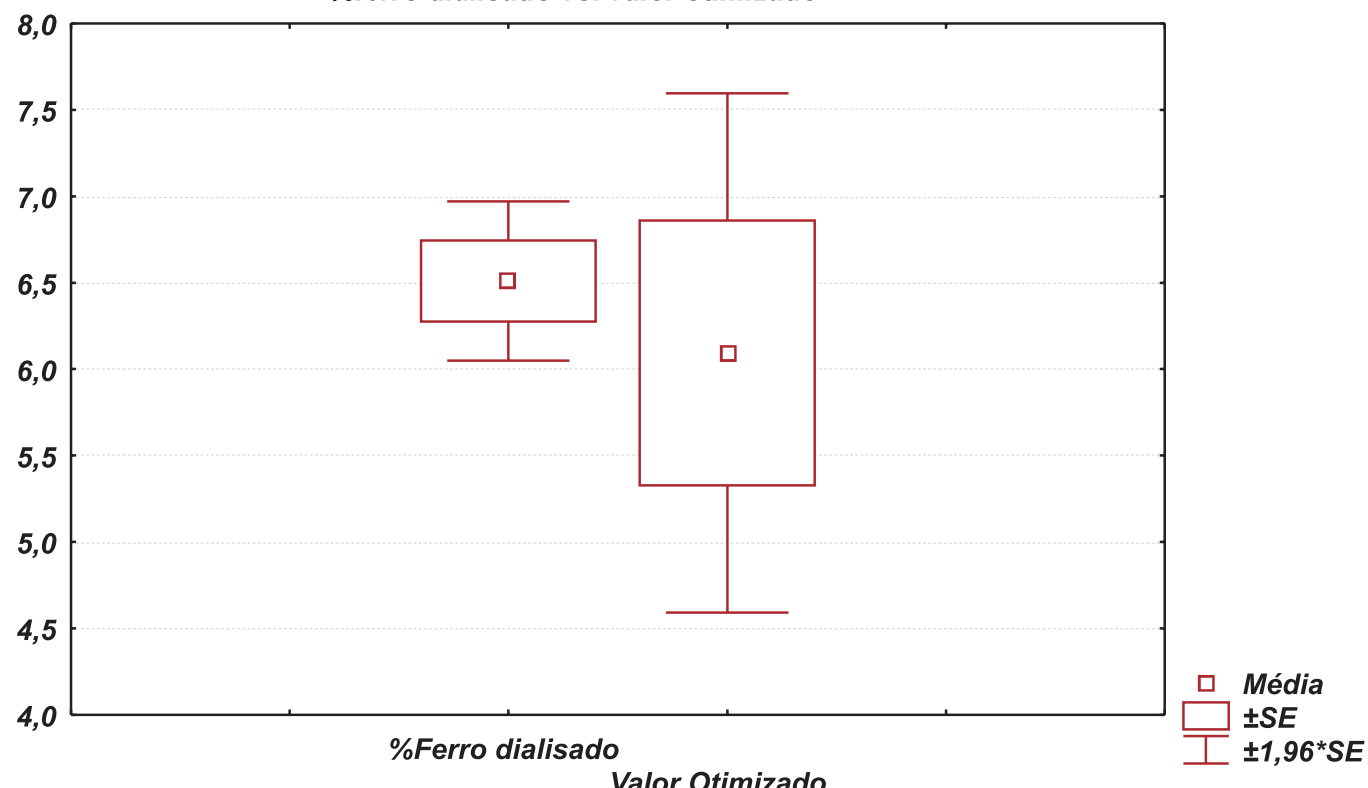

Figura 2S. Validação da dieta otimizada para o ferro dialisado

*e-mail: lubuenno@yahoo.com.br 


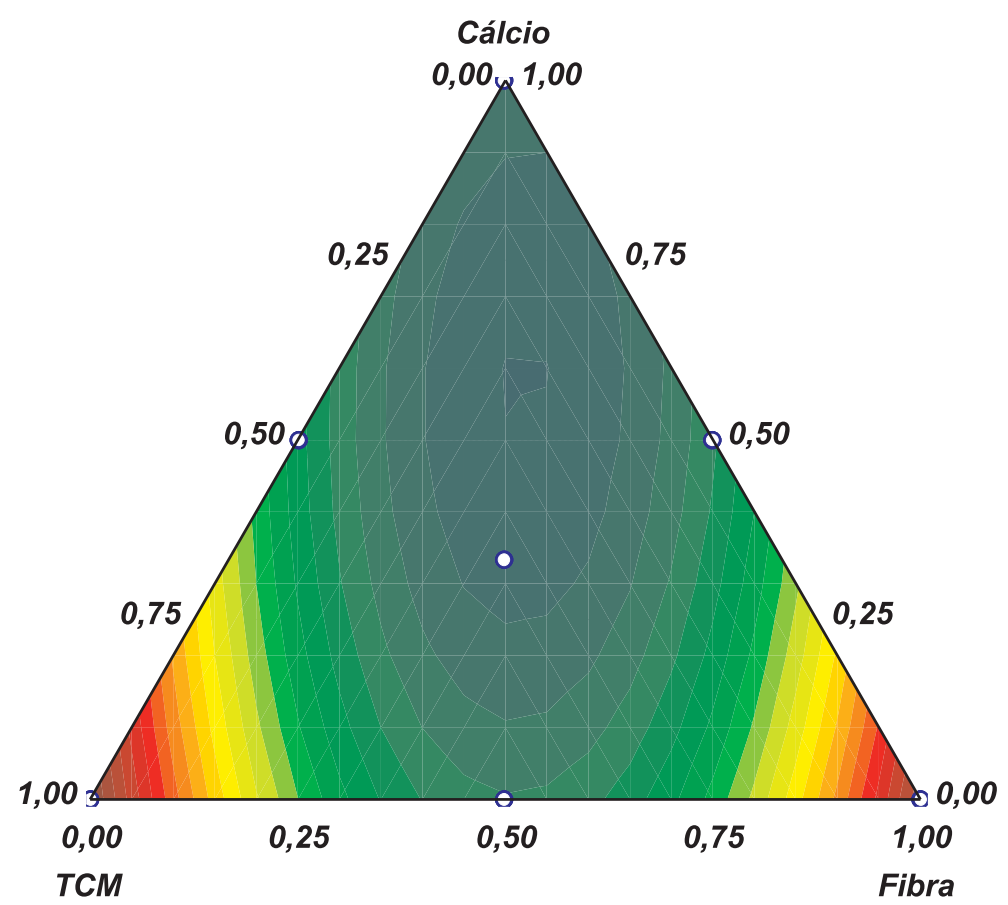

Figura 3S. Curvas de nível para a resposta do zinco dialisado

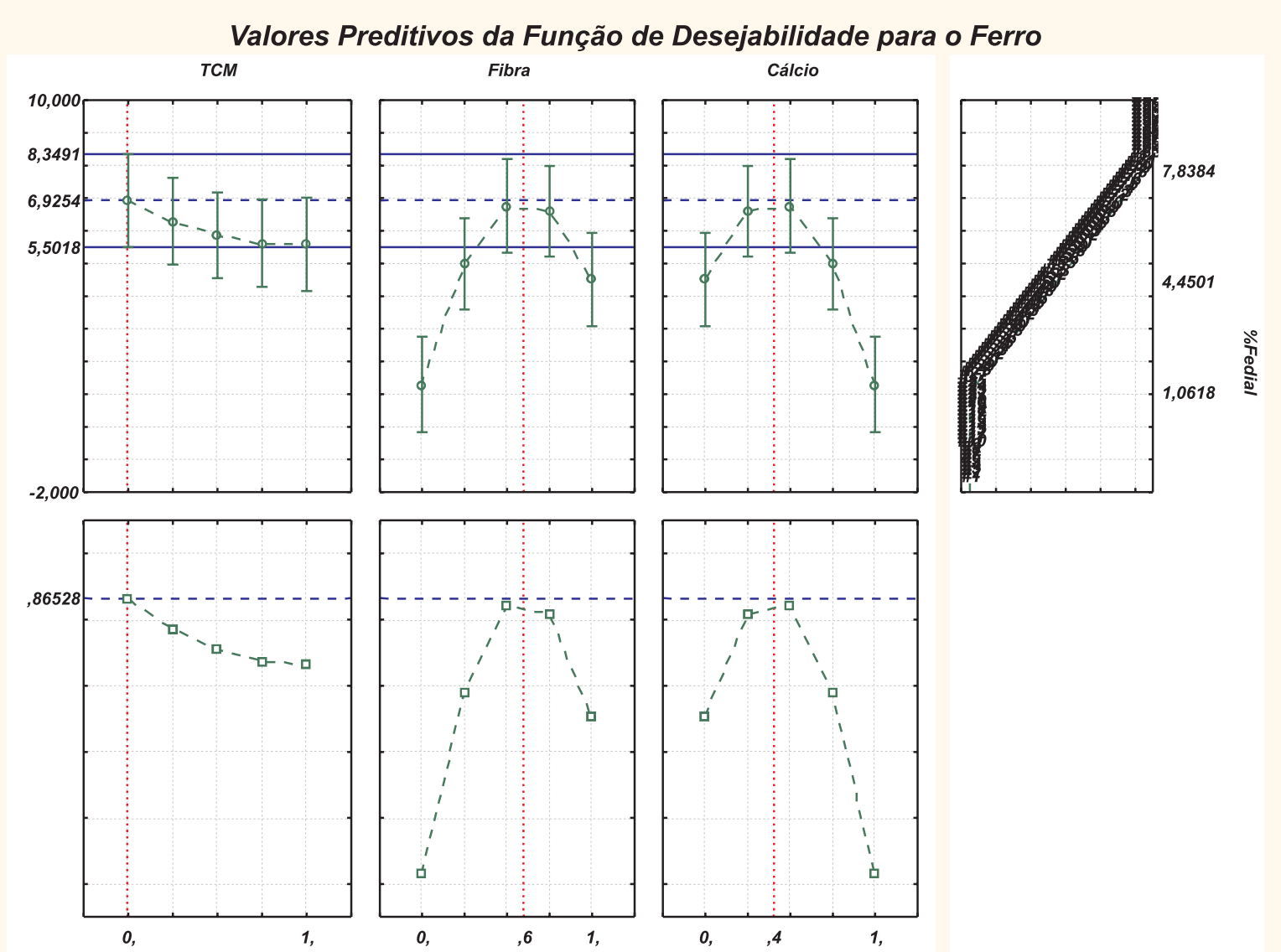

Figura 4S. Otimização de uma formulação de alimentação enteral para o ferro dialisado 
Valores Preditivos da Função de Desejabilidade para o Zinco

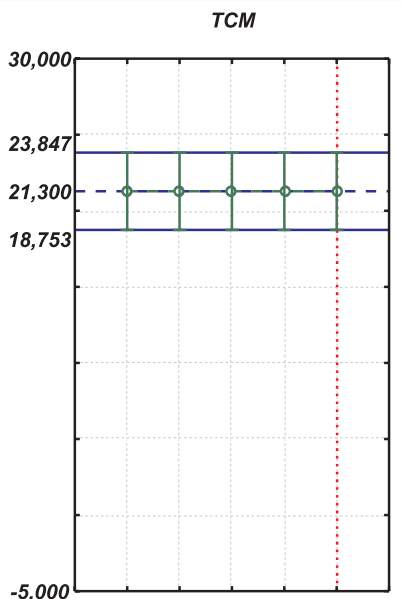

Fibra
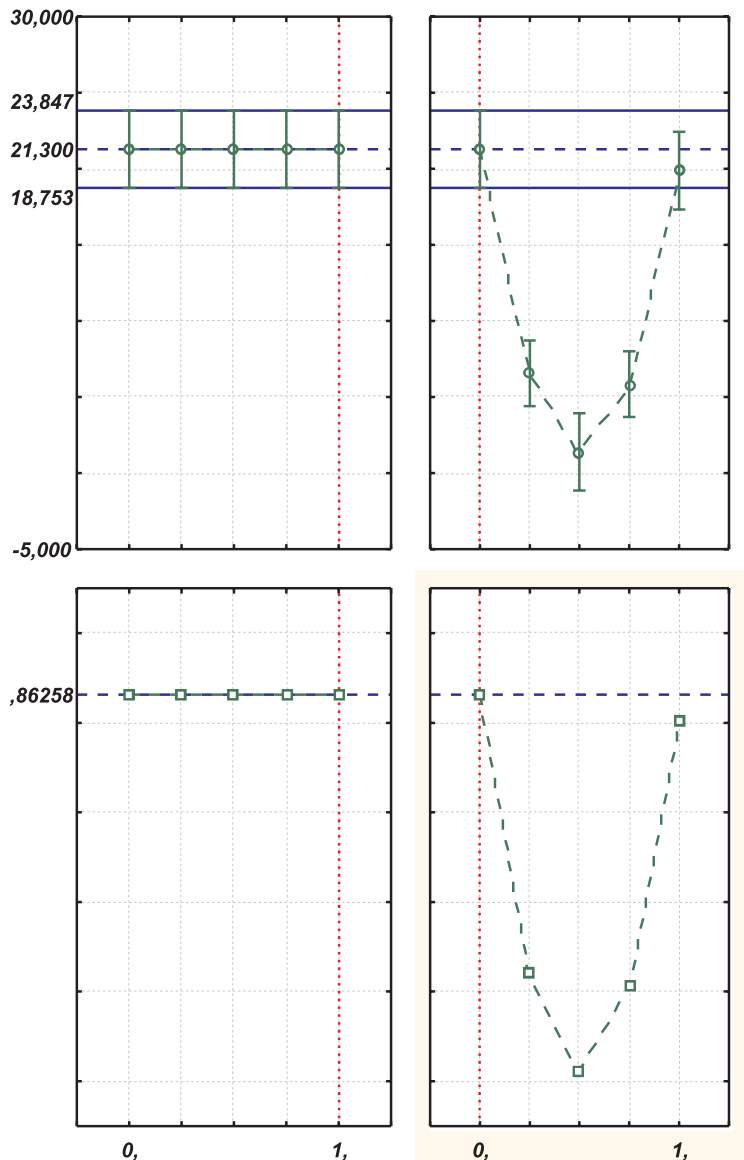

Cálcio
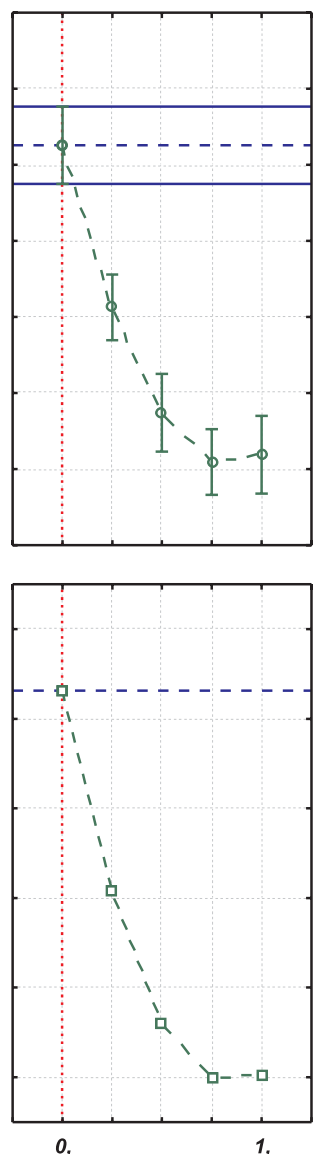

Desejabilidade

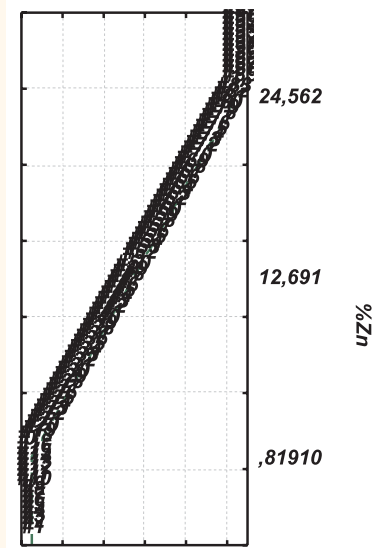

Figura 5S. Otimização de uma formulação de alimentação enteral para o zinco dialisado 